\title{
BMJ Open Delivery of compassionate mental health care in a digital technology- driven age: protocol for a scoping review
}

\author{
Gillian Strudwick, ${ }^{1,2,3}$ Timothy Zhang, ${ }^{2,4}$ Fiona Inglis, ${ }^{\circ}$ Sanjeev Sockalingam, ${ }^{5}$ \\ Mikayla Munnery, ${ }^{2,3}$ Brian Lo, ${ }^{2,3}$ Shuranjeet Singh Takhar, ${ }^{3}$ Rebecca Charow, ${ }^{\circledR 3,6}$ \\ David Wiljer ${ }^{3,6}$
}

\begin{abstract}
To cite: Strudwick G, Zhang T, Inglis $\mathrm{F}$, et al. Delivery of compassionate mental health care in a digital technologydriven age: protocol for a scoping review. BMJ Open 2019;9:e027989. doi:10.1136/ bmjopen-2018-027989

- Prepublication history and additional material for this paper are available online. To view these files, please visit the journal online (http://dx.doi. org/10.1136/bmjopen-2018027989).
\end{abstract}

Received 16 November 2018 Revised 29 March 2019 Accepted 17 June 2019

Check for updates

(C) Author(s) (or their employer(s)) 2019. Re-use permitted under CC BY-NC. No commercial re-use. See rights and permissions. Published by BMJ.

For numbered affiliations see end of article.

Correspondence to Dr Gillian Strudwick; gillian.strudwick@camh.ca

\section{ABSTRACT}

Introduction As digital technologies become an integral part of mental health care delivery, concerns have risen regarding how this technology may detract from health professionals' ability to provide compassionate care. To maintain and improve the quality of care for people with mental illness, there is a need to understand how to effectively incorporate technologies into the delivery of compassionate mental health care. The objectives of this scoping review are to: (1) identify the digital technologies currently being used among patients and health professionals in the delivery of mental health care; (2) determine how these digital technologies are being used in the context of the delivery of compassionate care and (3) uncover the barriers to, and facilitators of, digital technology-driven delivery of compassionate mental health care.

Methods and analysis Searches were conducted of five databases, consisting of relevant articles published in English between 1990 and 2019. Identified articles will be independently screened for eligibility by two reviewers, first at a title and abstract stage, and then at a full-text level. Data will be extracted and compiled from eligible articles into a data extraction chart. Information collected will include a basic overview of the publication including the article title, authors, year of publication, country of origin, research design and research question addressed. On completion of data synthesis, the authors will conduct a consultation phase with relevant experts in the field.

Ethics and dissemination Ethical approval is not required for this scoping review. With regards to the dissemination plan, principles identified from the relevant articles may be presented at conferences and an article will be published in an academic journal with study results. The authors also intend to engage interested mental health professionals, health professional educators and patients in a discussion about the study findings and implications for the future.

\section{INTRODUCTION}

While the medical field continues to benefit from advances in digital technology, it is important to consider the unintended consequences of these advances on health professional-patient interactions and the overall

\section{Strengths and limitations of this study}

- This scoping review is the first known to uncover and synthesise literature related to the influence of digital technology on the delivery of compassionate care in mental health contexts.

- An established and evidence-based scoping review framework was used to guide the development of the methodology, including an often overlooked sixth stage (consultation), in addition to the use of the newly published Preferred Reporting Items for Systematic review and Meta-Analysis-Scoping Review.

- As compassion is a difficult concept to define and measure, a possible limitation of this study is that relevant articles may have been inadvertently excluded due to search term limitations.

- A limitation of scoping reviews is that identified articles will not undergo a quality screening.

delivery of care. ${ }^{1}$ With digital technologies such as electronic health records (EHRs), patient portals and virtual/telehealth interventions being used to support care, ${ }^{2}$ some suggest that the increases in efficiency brought on by technology may detract from the delivery of patient-centred and compassionate care. ${ }^{3}$

In the last decade, there has been a growing interest in the use of digital technologies in mental health settings. ${ }^{2}$ Digital technology has been purported as a solution to addressing existing coordination of, and timely access to, mental health care. ${ }^{4-6}$ For example, there has been research demonstrating telemedicine interventions, like internet-based cognitive behavioural therapy (iCBT), can be as effective as traditional face-to-face therapies for treatment of disorders such as anxiety and depression, while requiring far fewer resources. ${ }^{4}$ iCBT and other behavioural intervention technologies, such as telephone, videoconferencing, web-based and mobile health interventions, 
have been shown to increase therapist reach and provide accessible mental health services to a broader range of patients and providers. ${ }^{45}$

Although a systematic review examining the use of technology in mental health settings has demonstrated benefits in improving communication among care team providers and overall patient care, the literature was limited to specific technologies (ie, EHRs) and did not specifically explore the effects on patient-provider relationships. ${ }^{6}$ Additionally, another systematic review of internet-based mental health interventions has shown that human-guided internet-based interventions, like iCBT, are often more effective than unguided self-help interventions. ${ }^{7}$ While it is generally unknown why human support is important specifically in internet-based therapies, a strong therapeutic alliance has been shown to be positively associated with programme adherence and treatment outcomes. ${ }^{4}$ Given the importance of this therapeutic alliance on mental health patient outcomes, ${ }^{89}$ there is a great need to advance our understanding of the relationship between digital technology use and compassionate care in mental health settings. More importantly, understanding how these technologies can be used to deliver compassionate care in mental health contexts is needed.

\section{Defining compassion}

The definition of compassion itself has seen multiple iterations across a variety of disciplines such as nursing, social work, psychology and medicine. ${ }^{1} 1011$ A working definition by the Oxford Handbook of Compassion Science suggests that compassion consists of distinct, yet subjective stepwise components, namely: (1) awareness of another's experience of suffering or need; (2) feeling 'moved'; (3) recognising this feeling as a response to the other's need; (4) making a judgement that the other is suffering and (5) engaging in a behaviour in an attempt to alleviate the suffering. ${ }^{12}$ Compassion should be treated as a fundamental element of effective health care, given that it has been cited as not only a necessary component in increasing patient's trust in their health professionals, but also a critical component of healing. ${ }^{13} 14$ Additionally, compassion is a different concept than sympathy and empathy since it is action-oriented and does not solely involve feelings of pity or sorrow for another.

\section{Compassion, technology and mental health care}

Patients who receive compassionate care are often able to more comfortably divulge information to a health professional, as they may feel emotionally safe and less anxious. ${ }^{1}$ The benefits of compassionate care also extend to health professionals. Strong human connections helps to prevent burnout and improve morale in both health professionals and trainees, which, in turn, may improve patient outcomes, creating a desirable feedback loop. ${ }^{1}$ However, several accounts outline how digital technologies have detracted from the meaningfulness of health professional-patient interactions. ${ }^{15-17}$ For example, with the emergence of EHRs steadily replacing hand-written documentation, patients are finding themselves forced to acclimatise to a screen, monitor or computer being the 'third party' during their interactions with health professionals. ${ }^{1}$ The changes in body language while distracted by a monitor, screen or computer may appear to convey a loss of concern and empathy, which could threaten the health professional-patient relationship. ${ }^{1}$ Technological 'distractions' may be more readily accepted in scenarios where the health professional needs to note a piece of objective information, such as a blood pressure reading; however, the context shifts when considering a mental health setting, where the relationship and interactions between patients and health professionals is often an integral part of assessment and treatment. ${ }^{15}$ Given the importance of a trusting relationship between a patient and a health professional in mental health, ${ }^{89}$ any degradation to interaction and communication by virtue of technology (or otherwise) may negatively impact patient outcomes. ${ }^{1} 18$

As digital technology usage within mental health care settings will only become more prevalent and complex in the future, ${ }^{2} 18$ there is a need to understand how technology can be appropriately incorporated into practice without sacrificing compassion and ultimately quality of care. It has been suggested that education is one avenue to support both technology integration alongside the promotion of compassionate care ${ }^{19}{ }^{20}$ Despite this, little can be done to improve the situation since there is a lack of knowledge surrounding: (1) how health professionals can properly leverage technology in compassionate ways and (2) the process of integrating technology into clinical practice interactions. Thus, a scoping review will be conducted to synthesise available literature regarding the current state of technology use, and how it is used in the delivery of compassionate mental health care. The objectives of this scoping review are to: (1) identify the digital technologies currently being used by patients and health professionals in the delivery of mental health care; (2) identify the relationships between digital health care and compassionate care in the mental health context and (3) uncover the barriers to, and facilitators of digital technology-driven delivery of compassionate mental health care. The overall purpose of conducting such a review is to initiate a conversation about if and how digital technologies can support or detract from the delivery of compassionate mental health care, and to identify pressing research needs on this topic for the future.

\section{METHODS AND ANALYSIS}

A scoping review methodology was chosen as it best suits the overall objectives of this study to inform gaps in evidence and clarify key concepts, particularly in a field of emerging evidence. ${ }^{21}$ This scoping review will be conducted based on the five-stage framework outlined by Arksey and O'Malley, ${ }^{22}$ and further developed by Levac, Colquhoun and O'Brien. ${ }^{23}$ Specifically, Arksey 
and O'Malley's optional sixth stage, 'consultations', will be incorporated in the scoping review. The Preferred Reporting Items for Systematic review and Meta-Analysis (PRISMA) flow diagram ${ }^{24}$ will also be used alongside the PRISMA-ScR (Scroping Review) checklist for scoping reviews. ${ }^{25}$ Article searches took place from September to December 2018, while data screening, extraction and synthesis will take place from January to May 2019 with the scoping review projected to be completed by the end of summer 2019. The six stages of the scoping review methodology are described below.

\section{Stage 1: identifying the research questions (RQs)}

In order to explore the relationship between digital technologies and compassionate mental health care, three RQs for the scoping review were determined as follows:

1. What existing digital technologies are most commonly used among patients and/or health professionals in the delivery of mental health care?

2. What are the relationships between digital technologies and compassionate mental health care? And, how are existing digital technologies being used among patients/health professionals in the delivery of compassionate mental health care?

3. What are the perceived barriers to and facilitators of using digital technology among patients and/or health professionals to deliver compassionate mental health care?

\section{Stage 2: identifying relevant studies \\ Eligibility criteria}

While all types of research studies (experimental, quantitative, qualitative, observational and so on) will be eligible for this review from any country of origin, only studies published in English will be included. Given the continuous advancement of digital technology, only studies published between 1990 and 2019 will be included given the timeframes in which many modern digital technologies such as EHR systems have been implemented. All formal mental health settings will be eligible (inpatient, outpatient, acute, clinics and so on) including clients of various age groups (adolescents, young adults, adult and senior populations). Eligible studies must address or provide information about at least one of the three stated RQs and include the use of digital technology in regards to mental health patient care. The interventions eligible include, but are not limited to: peer-to-peer support networks, self-help through usage of apps, blended or stepped care treatments and the training of health care professionals. The working definition for compassion will be that described in the introduction section of the protocol from the Oxford Handbook of Compassion Science. As it is difficult to have a strict criteria for compassion, we will also include articles that focus on the impact of technology on patient care, or are otherwise assessed as related to compassion by the research team. In the context of this review, digital technology will encompass all types of systems/applications for digital care (eg, EHRs), delivery of care modes and formats (eg, devices) and digital interfaces between individuals and the delivery of care (eg, patient portals). We will be explicitly excluding articles which focus on technologies being used in the context of measuring emotion, as well as medical devices such as blood pressure measuring equipment and imaging equipment.

\section{Search strategy}

The search strategy was developed collaboratively between the authors and an experienced research librarian, and includes searches of Medline, Medline In-Process and EPub Ahead of Print and PsycINFO through the Ovid interface, CINAHL (EBSCO) and Web of Science. Since the topic of this review is partially about patient interactions with health professionals, these databases were selected to capture the perspectives of various health professional disciplines, while incorporating diversity to avoid possible biases. Grey literature will not be included in the search. As part of the search strategy development, multiple iterations of the strategy were created and individually assessed for quality by using key, pre-identified articles as markers to ensure the desired elements were being captured. The search strategy also builds on previous work done by Wiljer $e t a t^{26}$ which examined definitions for compassion in the digital health care age. A search approach which incorporated elements of the search strategy from this previous review was undertaken for two main reasons. First, the concept of 'compassion' is difficult to fully articulate in predetermined search terms, so it is useful to leverage previous successful searches to improve how the concept of 'compassion' can be searched and captured in this review. Second, the working definition of technology for this review fully encompasses digital technologies, which was used as the definition of technology in the Wiljer et al review. ${ }^{26}$

Search terms being used include both subject headings and keywords associated with the concepts of compassionate care, technology and mental health care settings. Since the term 'compassion' may encompass many definitions based on context, interpretation and perspective, multiple synonyms and possible alternative terms for 'compassion' were included, such as empathy, patient-centred care and sense of rapport. Similarly, the concept of technology was designed to include systems (patient portals or EHRs), hardware (computers or mobile devices) and interventions (virtual or telehealth). An example of the complete search strategy developed for Medline is available in online supplementary 1 . This search strategy will be appropriately adapted and modified as required by the research librarian for execution across the other databases.

\section{Stage 3: study selection}

Further study selection will be done by assessing the fit of the identified articles from the initial search to the eligibility criteria. Two reviewers will independently screen the articles based on the eligibility criteria at a title and abstract 
Table 1 Data extraction table for the three research questions

\begin{tabular}{|c|c|}
\hline & Data to be abstracted \\
\hline \multirow[t]{8}{*}{ Article summary } & 1.1 Title \\
\hline & 1.2 Author(s) \\
\hline & 1.3 Year of publication \\
\hline & 1.4 Research design \\
\hline & 1.5 Country of publication \\
\hline & 1.6 Journal \\
\hline & 1.7 Citation \\
\hline & $\begin{array}{l}1.8 \mathrm{RQs} \text { addressed (1, } 2 \text { and/ } \\
\text { or } 3)\end{array}$ \\
\hline \multirow[t]{2}{*}{$\begin{array}{l}\text { RQ1: Digital technologies } \\
\text { in use }\end{array}$} & $\begin{array}{l}2.1 \text { Name of digital technology } \\
\text { in use }\end{array}$ \\
\hline & $\begin{array}{l}2.2 \text { General digital technology } \\
\text { category }\end{array}$ \\
\hline $\begin{array}{l}\mathrm{RQ2} \text { : Digital technology } \\
\text { usage methods/ } \\
\text { relationship to } \\
\text { compassionate care }\end{array}$ & $\begin{array}{l}3.1 \text { How digital technology is } \\
\text { being used and how it relates to } \\
\text { the delivery of compassionate } \\
\text { mental health care }\end{array}$ \\
\hline \multirow[t]{2}{*}{$\begin{array}{l}\text { RQ3: Barriers and } \\
\text { facilitators }\end{array}$} & $\begin{array}{l}\text { 4.1 Descriptors of barriers to } \\
\text { digital technology use }\end{array}$ \\
\hline & $\begin{array}{l}\text { 4.2 Descriptors of facilitators to } \\
\text { digital technology use }\end{array}$ \\
\hline
\end{tabular}

$R Q$, research question.

level, followed by a full-text screening. As a pilot test, the first 50 articles screened will be considered as a calibration and will not be included in the final reported percentage agreement and Cohen's kappa. If the reviewers do not reach an inter-rater reliability set at $80 \%$ percentage agreement and a Cohen's kappa of 0.60 , pilot testing will continue in intervals/batches of 50 citations until it is reached. During this period, the two reviewers will meet and thoroughly discuss any disagreements with the lead researcher to ensure consensus moving forwards. The calibration of both reviewers is especially important in the context of this review, as it allows for a second perspective on the subjective interpretation of what concepts may be used to describe compassion. Following the pilot testing period, any subsequent disagreements between the reviewers at either stage will be discussed on a case-by-case basis and resolved in consultation with the lead researcher as required.

Covidence (a literature review screening software recommended by Cochrane) ${ }^{27}$ will be used to filter duplicate articles, facilitate the aforementioned screening process and collect disagreement data between the two reviewers to calculate both the percentage agreement and Cohen's kappa. A PRISMA flow diagram for scoping reviews will also be used to visually report the search screening process. ${ }^{24}$

\section{Stage 4: data items and data collection process}

A chart (table 1) has been developed by the authors to efficiently extract and synthesise relevant data from the final eligible articles. Two members of the research team will have input into the validation of extracted data throughout the charting process, which will capture the following information categories:

1. Article summary: Information collected will include a basic overview of the publication including the article title, authors, year of publication, country of origin, research design and RQ addressed.

2. RQ1: The technologies that are currently being used by patients and health professionals in the delivery of mental health care will be recorded, and categorised by type.

3. RQ2: Information related to how digital technology is being used by patients and health professionals to specifically facilitate the delivery of compassionate mental health care will be described, as well as the relationship between the technology and compassionate mental health care.

4. RQ3: Identified barriers to, and facilitators of technology use by patients and health professionals in the delivery of compassionate mental health care will be listed.

Additional sub-items of interest may be included once the screening process begins. In accordance with scoping review methodology, each article's quality will not be assessed.

\section{Stage 5: synthesising and reporting the results}

To synthesise the results of the scoping review, both qualitative and quantitative methods will be applied depending on the RQ. Identified technologies for RQ1 will undergo a quantitative frequency analysis (descriptive statistics) with SPSS V.24 (IBM Released 2016; IBM SPSS Statistics for Windows, V.24.0), and summarised alongside a graphical representation. Conversely, a content analysis will be conducted on qualitative data obtained for RQ2 and RQ3 with the use of NVivo V.11 (QSR International Pty Ltd. Version 11, 2015), a qualitative data analysis software. $^{28}$ Key descriptors and concepts will be extracted and summarised in a table.

\section{Stage 6: consultation}

During stage 6 , the research team will conduct consultations with experts in the field. The objective of these consultations will be to validate any initial findings, identify further knowledge translation strategies and determine how findings from the review will be used to inform future research. Consultations will be done via either in-person meetings or through video teleconferences with up to 10 individuals from a diverse array of organisations that provide mental health service delivery. The research team will seek feedback from patients, their family members/care-partners, researchers who have published on the topic and mental health professionals. Key points from the consultations will be extracted, documented and summarised along with any changes resulting from the integration of the feedback. A more fulsome 
knowledge translation strategy will be determined based on these consultations.

\section{PATIENT AND PUBLIC INVOLVEMENT}

This scoping review protocol was developed without patient or public consultation. Patients were not involved in the writing or editing of this document. Although patients or the public were not initially involved in the development of the protocol, the authors intend to discuss the results of the review and solicit feedback and implications from patients for the next step of this work.

\section{ETHICS AND DISSEMINATION}

As this study is a type of literature review, ethical approval from the lead organisation's Research Ethics Board was not required. With regards to dissemination, the scoping review will be used to identify what is already known about the influence of technology on the delivery of compassionate care in a mental health setting. This will serve as a basis for multiple future research activities, such as uncovering patient and health care professionals' perceptions of how technology can be used to deliver compassionate mental health care. Given the continuous advancement and increasing presence of technology in health care, these findings will be extremely relevant and of use to nurses, doctors and other health professionals alike currently practicing in technology-laden mental health environments. As such, the cumulative results from these activities will be further translated directly to health professionals so they can be applied to their practice. This will be done through workshops with health professionals from a variety of organisations in mental health settings across Ontario, Canada.

Additionally, the findings of this scoping review will be submitted for presentation at the AMS Phoenix 'Bringing Compassion to Health Care' Conference, and may also be submitted to the 'Compassion in Action Health care Conference'. A manuscript resulting from this review, as well as all subsequent aforementioned research, will be submitted for publication in a relevant open-access journal to ensure the information has as large of a reach as possible. These activities are intended to maximise the knowledge translation of our findings by reaching the most relevant stakeholders both in Canada and beyond, allowing for the opportunity to both inform and receive feedback from key experts.

\footnotetext{
Author affiliations

${ }^{1}$ Campbell Family Mental Health Research Institute, Centre for Addiction and Mental Health, Toronto, Ontario, Canada

${ }^{2}$ Information Management Group, Centre for Addiction and Mental Health, Toronto,

Ontario, Canada

${ }^{3}$ Institute of Health Policy, Management and Evaluation, University of Toronto, Toronto, Ontario, Canada

${ }^{4}$ School of Public Health and Health Systems, University of Waterloo, Waterloo, Ontario, Canada

${ }^{5}$ Education Department, Centre for Addiction and Mental Health, Toronto, Ontario, Canada
}

${ }^{6}$ Education, Technology and Innovation, University Health Network, Toronto, Ontario, Canada

Acknowledgements The authors would like to thank Alicia Morgan and Jessica Kemp for their contributions to the development of this protocol. There were no patient or public contributions to the development of this protocol. The protocol has been registered though the Open Science Framework.

Contributors This scoping review was conceptualized and designed by GS and DW, with input from TZ. The search strategy was developed by Fl. A draft of the full manuscript was written initially by $\mathrm{TZ}$, and then GS. DW, FI, RC, MM, BL, SST and SS made contributions to the protocol writing. All authors have approved the final protocol.

Funding This work was supported by funds provided by the Associated Medical Services through the 2018 AMS Phoenix Fellowship program.

Competing interests None declared.

Patient consent for publication Not required.

Provenance and peer review Not commissioned; externally peer reviewed.

Open access This is an open access article distributed in accordance with the Creative Commons Attribution Non Commercial (CC BY-NC 4.0) license, which permits others to distribute, remix, adapt, build upon this work non-commercially, and license their derivative works on different terms, provided the original work is properly cited, appropriate credit is given, any changes made indicated, and the use is non-commercial. See: http://creativecommons.org/licenses/by-nc/4.0/.

\section{REFERENCES}

1. Post SG, Ng LE, Fischel JE, et al. Routine, empathic and compassionate patient care: definitions, development, obstacles, education and beneficiaries. J Eval Clin Pract 2014;20:872-80.

2. Mental Health Commission of Canada. E-mental health in Canada: transforming the mental health system using technology. $2014 \mathrm{http}: / /$ www.mentalhealthcommission.ca/English/system/files/private/ document/MHCC_E-Mental_Health-Briefing_Document_ENG.pdf

3. Canadian Medical Association. Health care transformation in Canada, 2010.

4. Pihlaja S, Stenberg JH, Joutsenniemi K, et al. Therapeutic alliance in guided internet therapy programs for depression and anxiety disorders - A systematic review. Internet Interv 2018;11:1-10.

5. Mohr DC, Burns MN, Schueller SM, et al. Behavioral intervention technologies: evidence review and recommendations for future research in mental health. Gen Hosp Psychiatry 2013;35:332-8.

6. Falconer E, Kho D, Docherty JP. Use of technology for care coordination initiatives for patients with mental health issues: a systematic literature review. Neuropsychiatr Dis Treat 2018;14:2337-49.

7. Baumeister $\mathrm{H}$, Reichler L, Munzinger $\mathrm{M}$, et al. The impact of guidance on Internet-based mental health interventions - A systematic review. Internet Interv 2014;1:205-15.

8. Kidd SA, Davidson L, McKenzie K. Common factors in community mental health intervention: a scoping review. Community Ment Health J 2017;53:627-37.

9. Howgego IM, Yellowlees P, Owen C, et al. The therapeutic alliance: the key to effective patient outcome? A descriptive review of the evidence in community mental health case management. Aust $N Z J$ Psychiatry 2003;37:169-83.

10. Radey M, Figley CR. The social psychology of compassion. Clin Soc Work J 2007.

11. Swanson KM. Nursing as informed caring for the well???being of others. Image J Nurs Scholarsh 1993.

12. Seppala E, Brown SL, Simon-Thomas E, et al. The Oxford Handbook of Compassion Science. Oxford University Press 2017.

13. Brodwin P. Everyday Ethics: Voices from the Front Line of Community Psychiatry. University of California Press 2013.

14. Clayton AR. How 'Person-Centered' care helped guide me toward recovery from mental illness. Health Aff 2013.

15. Strudwick G, Eyasu T. Electronic health record use by nurses in mental health settings: a literature review. Arch Psychiatr Nurs 2015;29:238-41.

16. Booth $\mathrm{N}$, Robinson $\mathrm{P}$, Kohannejad J. Identification of high-quality consultation practice in primary care: the effects of computer use on doctor-patient rapport. Inform Prim Care 2004;12:75-83.

17. Lanier C, Dominicé Dao M, Hudelson $\mathrm{P}$, et al. Learning to use electronic health records: can we stay patient-centered? A pre-post 
intervention study with family medicine residents. BMC Fam Pract 2017;18:1-11.

18. Mental Health Commission of Canada. Mental health, technology and you. Ottawa, Canada, 2017.

19. Johnston SC. Anticipating and Training the Physician of the Future. Acad Med 2018.

20. Associated Medical Services. Transformational trends in healthcare, 2017.

21. The Joanna Briggs Institute. The Joanna Briggs Institute Reviewers' Manual 2015 Methodology for JBI Scoping Reviews, 2015.

22. Arksey H, O'Malley L. Scoping studies: towards a methodological framework. Int J Soc Res Methodol 2005;8:19-32.

23. Levac D, Colquhoun H, O'Brien KK. Scoping studies: advancing the methodology. Implement Sci 2010;5:69.
24. Peters MD, Godfrey CM, Khalil H, et al. Guidance for conducting systematic scoping reviews. Int J Evid Based Healthc 2015;13:141-6.

25. Tricco AC, Lillie E, Zarin W, et al. prisma extension for scoping reviews (PRISMA-ScR): checklist and explanation. Ann Intern Med 2018;169:467.

26. Wiljer $\mathrm{D}$, Charow $\mathrm{R}$, Costin $\mathrm{H}$, et al. Defining compassion in the digital health age: protocol for a scoping review. BMJ Open 2019;9:e026338.

27. Veritas Health Innovation. Covidence systematic review software. Covidence, 2016.

28. Braun V, Clarke V. Using thematic analysis in psychology. Qual Res Psychol 2006;3:77-101. 